\title{
AVALIAÇÃO DA QUALIDADE DE VIDA NA INCONTINÊNCIA ANAL: validação do questionário FIQL (Fecal Incontinence Quality of Life)
}

\author{
Sonia Ahlaim Ibrahim YUSUF, José Marcio Neves JORGE, Angelita HABR-GAMA, \\ Desidério Roberto KISS e Joaquim GAMA RODRIGUES
}

RESUMO - Racional - A incontinência anal acarreta incapacitação física e psicológica, determinando impacto na qualidade de vida. Para quantificar esse impacto em nosso meio, não existem instrumentos específicos validados. Objetivos - Avaliar a qualidade de vida na incontinência anal, através da validação do questionário "Fecal Incontinence Quality of Life" (FIQL), que é composto por 29 questões distribuídas em 4 domínios: estilo de vida, comportamento, depressão e constrangimento, sua escala de pontuação varia de 1 a 4 com exceção das questões 1 e 4 que variam de 1 a 5 e 1 a 6, respectivamente. Material e Método - Após tradução e adaptação cultural, estudou-se a validação do instrumento através das propriedades de medida de reprodutibilidade e validade. Para a avaliação da reprodutibilidade aplicouse o questionário em 50 pacientes com incontinência anal por dois examinadores, sendo reaplicado por um dos examinadores após período de 7 a 10 dias. A validade construtiva foi testada através da comparação do FIQL e o SF-36, questionário genérico de qualidade de vida e entre o FIQL e um índice de incontinência anal. O índice de incontinência anal utilizado foi o de Jorge-Wexner, que varia de 0 (continência perfeita) a 20 (incontinência total). A validade discriminativa foi avaliada através da aplicação do FIQL em dois grupos controle: indivíduos voluntários hígidos e portadores de constipação intestinal. Resultados - Verificou-se que o FIQL apresentou correlação significativa com outros instrumentos (SF-36 e índice de incontinência) e que a qualidade de vida no portador de incontinência anal está comprometida em todos os domínios: estilo de vida: 2,4 comportamento: 2,0, depressão: 2,5 e constrangimento: 1,9, quando comparado com os indivíduos voluntários hígidos (3,9, 3,9, 4,1 e 4,0), e pacientes com constipação intestinal (3,7, 3,8, 3,6 e 3,8), respectivamente. Conclusão - O FIQL é útil para a avaliação da qualidade de vida na incontinência anal em nossa população.

DESCRITORES - Incontinência fecal. Qualidade de vida. Questionários.

\section{INTRODUÇÃO}

A perda do controle da evacuação determina incapacitação física e psicológica, resultando em perda da auto-estima e progressiva reclusão social. Estudos com crianças e indivíduos jovens demonstram que a incontinência anal resulta em impacto negativo na saúde mental e nos aspectos psicossociais ${ }^{(8,22)}$.

A incontinência anal onera o seu portador seja pelo custo de protetores das vestes, ou por demandar pessoal especializado, principalmente aos idosos institucionalizados, que muitas vezes se encontram expostos à infecção urinária e à dermatite perineal ${ }^{(6,39)}$.

A real prevalência da incontinência anal é desconhecida, já que freqüentemente a abordagem do sintoma é dificultada em virtude do constrangimento ${ }^{(9,25)}$. Embora esse distúrbio da evacuação possa acometer qualquer faixa etária, sua incidência é maior no sexo feminino, provavelmente devido a complicações no parto vaginal e a maior prevalência, neste grupo, da constipação intestinal com esforço evacuatório crônico ${ }^{(14,26,41)}$.
Qualquer que seja a etiologia ou o grupo acometido, o convívio social para esses pacientes é penoso, resultando em importante impacto no bem-estar e em sua qualidade de $v_{i d a}{ }^{(38,40)}$. Entretanto, a percepção da gravidade desses aspectos, resultantes da incontinência anal, é muito subjetiva e os testes objetivos não revelam relação direta entre o grau de incontinência anal e o comprometimento por ela acarretado.

Nos últimos anos, o resultado das intervenções médicas passou a ser avaliado, também, por meio de variáveis subjetivas que incorporam a percepção do indivíduo em relação ao seu bem-estar e expectativas de vida, especialmente nas áreas de domínios físico, social, emocional e ocupacional ${ }^{(4,12,13)}$. Com essa finalidade, vários instrumentos ou questionários têm sido desenvolvidos para a avaliação de doenças crônicas e especificamente na avaliação da incontinência anal, recentemente, foi proposto o FIQL - "Fecal Incontinence Quality of Life"(34), composto por 29 questões distribuídas em 4 domínios. Os domínios ou escalas representam grupos de

Disciplina de Coloproctologia do Departamento de Gastroenterologia da Faculdade de Medicina da Universidade de São Paulo, SP.

Endereço para correspondência: Dr. José Márcio Neves Jorge - Alameda Rio Claro, 95 - apt. 122 - 01332-010 - São Paulo, SP. 
itens ou questões que abordam o mesmo aspecto referente à qualidade de vida. Os domínios do FIQL e suas respectivas questões são: 1. estilo de vida: composto por 10 itens, Q2 (a, b, c, d, e, g, h), Q3 (b, 1, m); 2. comportamento: composto por 9 itens, Q2 (f, i, j, k, m), Q3 (c, h, j, n); 3. depressão: composto por 7 itens, Q1, Q3 (d, f, g, i, k), Q4; e 4. constrangimento: composto por 3 itens Q2 (1), Q3 (a, e). A pontuação dos itens do questionário varia de 1 a 4 , com exceção das questões 1 e 4 (Figura 1).

Para que um questionário seja considerado adequado e validado ele deve ser: confiável, reprodutível, sensível às alterações ou discriminações e ter validade demonstrada. A constatação da falta de questionário validado para avaliação da qualidade de vida na incontinência anal em nosso meio, motivou a realização do processo de validação do instrumento FIQL no presente estudo.

\section{PACIENTES E MÉTODOS}

Por ter sido concebido em idioma inglês, o questionário foi submetido a processo de tradução e adaptação cultural de acordo com recomendações internacionais antes de se iniciar o processo de validação ${ }^{(16)}$.

\section{Tradução}

O questionário FIQL original foi traduzido por dois professores da língua inglesa para o português. As versões foram comparadas entre si por um grupo multidisciplinar composto por dois médicos, uma enfermeira e duas psicólogas e, através de um consenso, foi originada a primeira versão do questionário em português. Essa versão foi traduzida para o inglês por dois professores de inglês de nacionalidade americana. Desta vez o grupo multidisciplinar avaliou todas as versões, originando-se a segunda versão do questionário em português, que foi utilizada para o processo de adaptação cultural.

FIGURA 1 - Versão final do questionário FIQL na língua portuguesa

Questão 1. Em geral você diria que sua saúde é:

Excelente () Muito boa () Boa () Regular ( ) Ruim ()

Questão 2. Para cada um dos itens abaixo, por favor, indique, marcando um X na coluna correspondente a quanto tempo o item abaixo o preocupa devido à perda de fezes

Se qualquer um dos itens lhe preocupa por outras razões que não pela perda de fezes, marque a alternativa "Nenhuma das respostas"

\begin{tabular}{|c|c|c|c|c|c|}
\hline & $\begin{array}{c}\text { Muitas } \\
\text { vezes }\end{array}$ & $\begin{array}{c}\text { Algumas } \\
\text { vezes }\end{array}$ & $\begin{array}{c}\begin{array}{c}\text { Poucas } \\
\text { vezes }\end{array} \\
\end{array}$ & $\begin{array}{c}\text { Nenhuma } \\
\text { vez }\end{array}$ & $\begin{array}{c}\text { Nenhuma das } \\
\text { respostas }\end{array}$ \\
\hline \multicolumn{6}{|l|}{ Devido à perda de fezes: } \\
\hline a. Tenho medo de sair & 1 & 2 & 3 & 4 & \\
\hline b. Evito visitar amigos ou parentes & 1 & 2 & 3 & 4 & \\
\hline c. Evito passar a noite longe de casa & 1 & 2 & 3 & 4 & \\
\hline d. É difícil para eu sair e fazer coisas como ir ao cinema ou à igreja & 1 & 2 & 3 & 4 & \\
\hline e. Evito comer antes de sair de casa & 1 & 2 & 3 & 4 & \\
\hline f. Quando estou fora de casa tento ficar sempre que possível próximo ao banheiro & 1 & 2 & 3 & 4 & \\
\hline g. É importante eu planejar o que vou fazer de acordo com o meu funcionamento intestinal & 1 & 2 & 3 & 4 & \\
\hline h. Evito viajar & 1 & 2 & 3 & 4 & \\
\hline i. Fico preocupado em não ser capaz de chegar ao banheiro em tempo & 1 & 2 & 3 & 4 & \\
\hline j. Sinto que não tenho controle do meu intestino & 1 & 2 & 3 & 4 & \\
\hline k. Não consigo controlar minha evacuação a tempo de chegar ao banheiro & 1 & 2 & 3 & 4 & \\
\hline 1. Perco fezes sem perceber & 1 & 2 & 3 & 4 & \\
\hline $\mathrm{m}$. Tento evitar a perda de fezes, ficando próximo ao banheiro & 1 & 2 & 3 & 4 & \\
\hline
\end{tabular}

Questão 3. Devido a sua perda de fezes indique até quanto o problema o incomoda. Se qualquer dos itens abaixo o preocupa por outras razões, marque a alternativa "Nenhuma das respostas"

\begin{tabular}{|c|c|c|c|c|c|}
\hline & Muitas vezes & Algumas vezes & Poucas vezes & Nenhuma vez & $\begin{array}{c}\text { Nenhuma das } \\
\text { respostas }\end{array}$ \\
\hline \multicolumn{6}{|l|}{ Devido à perda de fezes: } \\
\hline a. Fico envergonhado & 1 & 2 & 3 & 4 & \\
\hline b. Não posso fazer muitas coisas que quero fazer & 1 & 2 & 3 & 4 & \\
\hline c. Fico preocupado em perder fezes & 1 & 2 & 3 & 4 & \\
\hline d. Sinto-me deprimido. & 1 & 2 & 3 & 4 & \\
\hline e. Fico preocupado se outras pessoas sentem cheiro de fezes em mim & 1 & 2 & 3 & 4 & \\
\hline f. Acho que não sou uma pessoa saudável & 1 & 2 & 3 & 4 & \\
\hline g. Tenho menos prazer em viver & 1 & 2 & 3 & 4 & \\
\hline h. Tenho relação sexual com menor freqüência do que gostaria & 1 & 2 & 3 & 4 & \\
\hline i. Sinto-me diferente das outras pessoas & 1 & 2 & 3 & 4 & \\
\hline j. Sempre estou pensando na possibilidade de perder fezes. & 1 & 2 & 3 & 4 & \\
\hline k. Tenho medo de ter sexo & 1 & 2 & 3 & 4 & \\
\hline 1. Evito viajar de carro ou ônibus & 1 & 2 & 3 & 4 & \\
\hline m. Evito sair para comer & 1 & 2 & 3 & 4 & \\
\hline n. Quando vou a um lugar novo, procuro saber onde está o banheiro & 1 & 2 & 3 & 4 & \\
\hline
\end{tabular}

n. Quando vou a um lugar novo, procuro saber onde está o banheiro

Questão 4. Durante o mês passado, eu me senti tão triste, desanimado ou tive muitos problemas que me fizeram pensar que nada valia a pena

1. ( ) Extremamente. A ponto de quase desistir

2. ( ) Muitas vezes

3. ( ) Com frequiência

4. ( ) Algumas vezes - o suficiente para me preocupar (incomodar)

5. () Poucas vezes

6. () Nenhuma vez 


\section{Adaptação Cultural}

O questionário foi aplicado a um grupo de 20 pacientes com incontinência anal, selecionados aleatoriamente no ambulatório de fisiologia do cólon reto e ânus da Disciplina de Coloproctologia da Faculdade de Medicina da Universidade de São Paulo, SP. A cada questão da segunda versão do questionário acrescentou-se a opção "não aplicável" (N/A), a fim de se identificar quais questões seriam culturalmente não-compatíveis, ou até mesmo não compreendidas por nossa população. As questões que apresentaram mais de $15 \%$ de resposta "não aplicável" foram selecionadas e novamente redigidas pelo grupo multidisciplinar, procurando-se manter o mesmo conceito $^{(5,11)}$.

Outros 20 pacientes incontinentes foram selecionados, aos quais foi aplicada a terceira versão do questionário, observando-se que desta vez nenhuma questão apresentou resposta "não aplicável" acima de $15 \%$, considerando-se a adaptação cultural completa. Essa versão final do questionário foi utilizada no processo de validação (Figura 1).

\section{Validação}

As propriedades de medida estudadas foram a reprodutibilidade e a validade.

\section{Reprodutibilidade}

A reprodutibilidade da versão final do FIQL foi testada por meio de três entrevistas. Participaram desta fase 50 pacientes portadores de incontinência anal de etiologias diversas, sem tratamento prévio do sintoma e que não participaram da etapa de adaptação cultural.

A reprodutibilidade entre examinadores diferentes foi avaliada através de entrevistas realizadas pelos examinadores A e B no mesmo dia, com o mesmo paciente, com intervalo de 30 minutos entre uma e outra. O examinador A reavaliou todos os pacientes, após período de 7 a 10 dias, com a finalidade de se comparar os resultados obtidos pelo mesmo examinador em tempos diferentes. A média obtida entre as três entrevistas foi utilizada na etapa de validade.

\section{Validade}

Foram testadas a validade de construção e discriminativa.

Para a avaliação da validade de construção, os pacientes incontinentes responderam também ao índice de incontinência anal ${ }^{(22)} \mathrm{e}$ ao questionário genérico conhecido como Short-Form $36^{(45)}$, validado em nosso meio por CICONELLI ${ }^{(7)}$. Os resultados obtidos foram correlacionados com os do FIQL.

A validade discriminativa foi avaliada aplicando-se o FIQL em 30 pacientes com constipação intestinal e 30 indivíduos voluntários hígidos. Nesta etapa, a questão $n^{\circ} 2$ "devido à sua incontinência anal", foi modificada para "devido à sua constipação", para os pacientes constipados, e "de acordo com o seu estado de saúde", para os voluntários hígidos. Os respectivos resultados foram comparados aos obtidos com a média final da reprodutibilidade.

\section{Análise Estatística}

Foram utilizados o coeficiente de correlação intraclasse, análise de variância (ANOVA), o coeficiente de Pearson e teste $t$ de Student. O índice de significância estabelecido foi 0,05 .

\section{RESULTADOS}

\section{Adaptação Cultural}

Durante o processo de adaptação cultural, três questões pertencentes ao domínio 1 - estilo de vida - necessitaram ser modificadas: ao item 2 b acrescentou-se a palavra "parentes" visto que devido ao constrangimento, $40 \%$ dos pacientes visitavam apenas seus parentes. $\mathrm{O}$ item $2 \mathrm{~g}$, inicialmente traduzido como "é importante eu planejar o que eu vou fazer de acordo com o meu funcionamento intestinal”, mostrou-se incompreensível a $60 \%$ dos entrevistados, sendo modificado para "dependo do funcionamento do meu intestino para planejar o que vou fazer durante o dia". O item 31 foi modificado para "evito viajar de carro ou ônibus", já que $100 \%$ da população entrevistada não viajava de avião ou de trem.

Outras modificações foram: as alternativas "concordo plenamente", "concordo parcialmente", "discordo parcialmente" e "discordo plenamente" foram substituídas pelas alternativas "muitas vezes", "algumas vezes", "poucas vezes" e "nenhuma vez", respectivamente. A frase "indique em que até extensão o problema incomoda" foi modificada para "indique até quanto o problema o incomoda".

O tempo médio de aplicação do questionário foi de 13 (10 a 15) minutos.

\section{Validação}

Características sócio-demográficas - A população de pacientes incontinentes foi em sua maioria, constituída pelo sexo feminino 37 (74\%). Quanto à ocupação $10(20 \%)$ paciente estavam desempregados, dos quais 6 (12\%), atribuíram à incontinência a causa do desemprego. Quanto ao nível de escolaridade 44 (88\%) eram alfabetizados (Tabela 1).

TABELA 1 - Distribuição quanto ao sexo, nível de escolaridade e ocupação dos 50 pacientes com incontinência anal

\begin{tabular}{ll}
\hline Variável & $\mathbf{n}(\%)$ \\
\hline Sexo & $37(74 \%)$ \\
feminino & $13(26 \%)$ \\
masculino & $6(12 \%)$ \\
\hline Nível de escolaridade & $44(88 \%)$ \\
$\quad$ não alfabetizados & \\
$\quad$ alfabetizados & $40(80 \%)$ \\
\hline Ocupação & $10(20 \%)$ \\
empregados & \\
desempregados & \\
\hline
\end{tabular}

O tempo médio obtido de duração da incontinência anal foi de 10,2 anos.

A média de idade foi 52,8 anos, sendo a idade mínima de 15 e a máxima de 75 anos.

Reprodutibilidade - Os resultados obtidos para a reprodutibilidade intra- e interexaminadores mostraram concordância significativa em todos os domínios do questionário e estão demonstrados nas Tabelas 2 e 3.

TABELA 2 - Coeficientes intraclasse obtidos pelo mesmo examinador

\begin{tabular}{lcc}
\hline Variável & Média \pm desvio-padrão & $\begin{array}{c}\text { Coeficiente de correlação } \\
\text { intraclasse }\end{array}$ \\
\hline Estilo de vida & $2,36 \pm 1,23$ & $0,934(93,4 \%)$ \\
Comportamento & $2,28 \pm 1,15$ & $0.938(93,8 \%)$ \\
Depressão & $2,19 \pm 1,31$ & $0,957(95,7 \%)$ \\
Constrangimento & $1,84 \pm 1,03$ & $0,929(92,9 \%)$ \\
\hline$P<0,001$
\end{tabular}

$P<0,001$

TABELA 3 - Coeficientes intraclasse obtidos entre examinadores diferentes

\begin{tabular}{lcc}
\hline Variável & Média \pm desvio-padrão & Coeficiente de correlação intraclasse \\
\hline Estilo de vida & $2,34 \pm 1,25$ & $0,944(94,4 \%)$ \\
Comportamento & $2,25 \pm 1,17$ & $0.973(97,8 \%)$ \\
Depressão & $2,23 \pm 1,35$ & $0,957(95,7 \%)$ \\
Constrangimento & $1,95 \pm 1,09$ & $0,969(96,9 \%)$ \\
\hline
\end{tabular}




\section{Validade}

Validade construtiva - Observou-se correlação inversa entre os valores totais do FIQL e o índice de incontinência anal, isto é, pacientes com incontinência anal mais grave (índices mais elevados), apresentaram piores índices de qualidade de vida (valores mais baixos) $(P<0,001)$ (Gráfico 1). Em $11(22 \%)$ casos, no entanto, não se observou correlação entre os valores de FIQL e os do índice de incontinência anal.

GRÁFICO 1 - Correlação entre o índice de incontinência anal e o FIQL

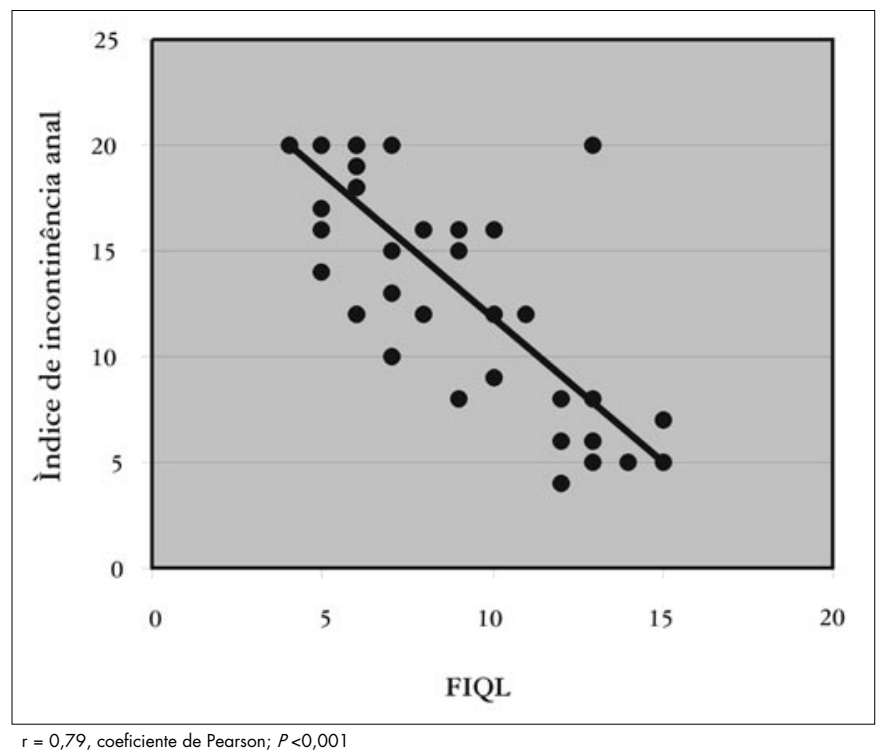

$r=0,79$, coeficiente de Pearson; $P<0,001$

$\mathrm{Na}$ análise da correlação entre os dois questionários de qualidade de vida - específico (FIQL) e genérico (SF-36) - observou-se correlação significativa entre todos os domínios do FIQL e do SF-36 (coeficiente 0,754 a 0,$556 ; P<0,01$ ), exceto o domínio dor (coeficiente $=0,103$; $P=$ NS) (Tabela 4).

TABELA 4 - Correlação dos resultados obtidos através da aplicação do FIQL com os resultados obtidos com a aplicação do Shortform 36

\begin{tabular}{lcccc}
\hline Variável & $\begin{array}{c}\text { Estilo de } \\
\text { vida }\end{array}$ & Comportamento & Depressão & Constrangimento \\
\hline Estado geral da saúde &, 65 &, 64 &, 66 &, 65 \\
Aspectos físicos &, 74 &, 62 &, 69 &, 64 \\
Aspectos sociais &, 66 &, 70 &, 61 &, 68 \\
Capacidade funcional &, 75 &, 64 &, 63 &, 66 \\
Vitalidade &, 65 &, 64 &, 70 &, 65 \\
Saúde mental &, 73 &, 71 &, 72 &, 65 \\
Aspectos emocionais &, 71 &, 55 &, 74 &, 72 \\
Dor** &, 08 &, 11 &, 11 &, 15 \\
\hline$P<0,01 \quad * * P=N S$, coeficiente de correlação de Pearson & &
\end{tabular}

Validade discriminativa - Verificou-se que a qualidade de vida no portador de incontinência anal está comprometida em todos os domínios estudados do FIQL: estilo de vida: 2,4, comportamento: 2,0, depressão: 2,5 , constrangimento: 1,9 e total: 8,4 , quando comparado com os indivíduos voluntários hígidos (3,9, 3,9, 4,1, 4,0 e 15,9), e pacientes com constipação intestinal $(3,7,3,8,3,6,3,8$ e 14,9), respectivamente $(P<0,05)$ (Gráfico 2).

GRÁFICO 2 - Comparação das médias obtidas entre os três grupos, incontinentes, constipados e voluntários hígidos ao ser aplicado o FIQL

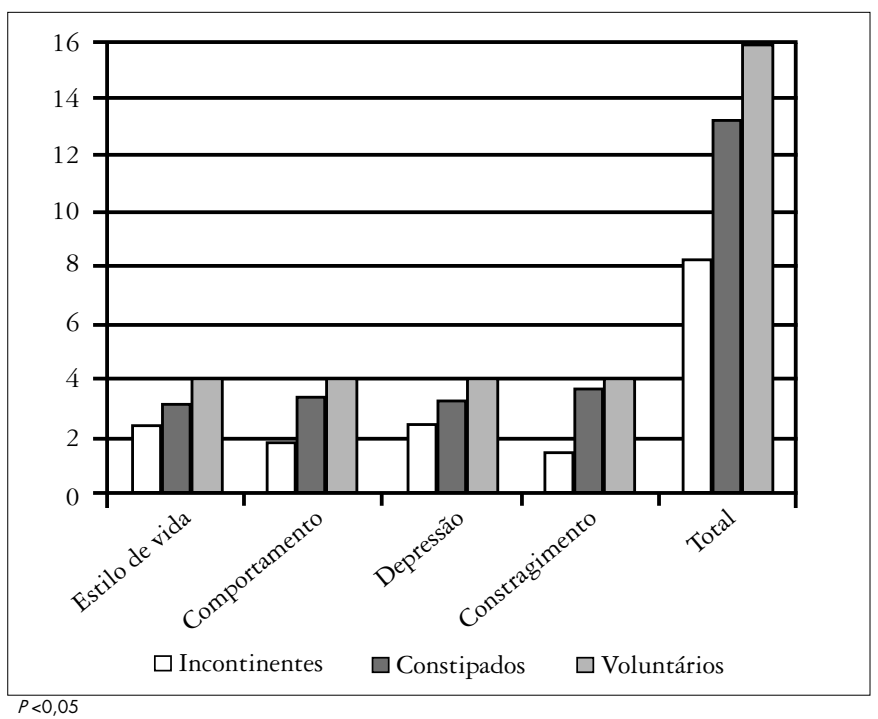

\section{DISCUSSÃO}

Existe na literatura uma escassez de métodos objetivos e específicos de medida para a abordagem do impacto na qualidade de vida nos pacientes com incontinência anal. Idealmente, a avaliação da qualidade de vida deve ser feita através de métodos quantitativos e reprodutíveis. Sobretudo, a seleção de um instrumento deve ser feita considerando-se a sua proposta, a clareza de seus componentes, a definição da populaçãoalvo e a doença para a qual essas medidas foram desenvolvidas. Além disso, o instrumento deve ser de fácil aplicação, de fácil compreensão e ter tempo de administração apropriado ${ }^{(2,43)}$.

Apesar de se encontrarem na literatura outros instrumentos específicos na incontinência anal, tais como o "Fecal Incontinence Questionnaire"(33) e "The Fecal Incontinence Severity Index"(35), o FIQL foi utilizado no presente estudo por se tratar de instrumento específico de linguagem simples, com tempo de aplicação viável, tendo recebido crescente aceitação na literatura ${ }^{(24,26,27,29,36)}$. A maioria dos instrumentos utilizados para avaliação da qualidade de vida, incluindo o FIQL, foi originalmente proposta no idioma inglês.

Atualmente, é consenso que um instrumento de avaliação de qualidade de vida deva, após processo de tradução e adaptação cultural cuidadoso, ter suas propriedades de medida testadas num contexto cultural específico, já que cada grupo social tem suas próprias crenças, atitudes, costumes, comportamento e hábitos ${ }^{(32,42)}$.

A importância da tradução e adaptação fez-se notar neste estudo, pelas mudanças necessárias para o melhor entendimento do questionário 
pelo entrevistado, como exemplo as alternativas "concordo plenamente," "concordo parcialmente," "discordo parcialmente" e "discordo plenamente" foram substituídas pelas alternativas "muitas vezes", "algumas vezes," “ poucas vezes" e "nenhuma vez," respectivamente, pois julgou-se que haveria dificuldade no discernimento entre "concordo parcialmente" e "discordo parcialmente". Quanto à reavaliação do peso dos índices, não houve necessidade de mudanças, uma vez que houve boa aceitação pela população estudada da pontuação preestabelecida para os itens do questionário.

A confiabilidade de um questionário deve ser avaliada através das propriedades de medida do instrumento, reprodutibilidade e validade ${ }^{(3,}$, ${ }^{44)}$. O bom instrumento de avaliação produz resultados iguais ou semelhantes, em duas ou mais aplicações no mesmo paciente, desde que seu estado clínico não tenha sido alterado(20)

Durante a aplicação do FIQL, a reprodutibilidade intra-examinador e interexaminador variou de 0,929 a 0,957 e de 0,944 a 0,969 , respectivamente, demonstrado correlação estatisticamente significativa para todos os domínios: estilo de vida, comportamento, depressão e constrangimento $(P<0,001)$.

A validade é a propriedade do instrumento em medir, da melhor forma possível, o fenômeno de interesse. Ressalta-se que os questionários de qualidade de vida, embora estudem a percepção da saúde do ponto de vista do paciente nos aspectos social, físico, emocional e psicológico, podem se correlacionar bem com medidas tradicionais utilizadas na avaliação do paciente ${ }^{(31)}$.

$\mathrm{Na}$ análise da correlação entre os dois questionários de qualidade de vida, observou-se que todos os domínios do questionário específico FIQL e os do questionário genérico, exceto o domínio dor (coeficiente $=0,103 ; P=\mathrm{NS})$, apresentaram boa correlação estatística entre si, pois o coeficiente variou de 0,754 a $0,556(P<0,01)$. É provável que a falta de correlação do domínio dor deva-se à ausência desse sintoma no doente portador de incontinência anal.

Com os resultados obtidos com a aplicação dos dois questionários, observou-se que o maior impacto ocorre nos aspectos psíquicos e funcionais da qualidade de vida do indivíduo. Resultados semelhantes são relatados por KOLOSKI et al. ${ }^{(23)}$ ao estudarem o impacto dos distúrbios gastrointestinais na qualidade de vida em 4.500 pacientes, dos quais 157 eram acometidos de incontinência anal.

JOHANSON e LAFFERTY ${ }^{(21)}$, em 1996, mostraram em estudo sobre qualidade de vida na incontinência anal, que $11 \%$ dos seus pacientes reduziram suas atividades no trabalho, devido ao sintoma. Observouse no presente estudo, que $6 \%$ da população atribuiu o desemprego à incontinência anal, seja pelo baixo rendimento relatado, justificado pela necessidade de interromper o trabalho para trocar a proteção das vestes várias vezes, seja pela dificuldade do convívio social.

HUPPE et al. ${ }^{(19)}$, em estudo sobre aspectos psicossociais da incontinência anal, observaram que os aspectos mais comprometidos foram a sexualidade e o desempenho do indivíduo no emprego. $\mathrm{Na}$ presente série verificou-se que $58 \%$ dos pacientes suspenderam sua atividade sexual, enquanto $14 \%$ somente diminuíram a freqüência dessa atividade, o que determinou a baixa pontuação nos domínios comportamento e depressão.
Nesta casuística, $68 \%$ dos doentes portadores de incontinência anal passaram a apresentar depressão após o início dos sintomas, entre os quais, seis evoluíram em idéias suicidas e um necessitou de internação psiquiátrica. Ressalte-se que os pacientes referiram espontaneamente ter idéias suicidas ao serem questionados sobre a presença de desânimo, tristeza ou nervosismo no último mês. Este fato poderia sugerir a necessidade da inclusão de questões que abordem também este aspecto, tais como as encontradas nas escalas de avaliação da depressão de BECK e STEER ${ }^{(1)}$ e HAMILTON ${ }^{(18)}$.

Ainda que em sua maioria os pacientes tivessem apresentado resultados do índice de incontinência anal correspondentes ao índice de qualidade de vida, em $22 \%$ dos casos isso não ocorreu. Entre esses, observou-se em cinco casos (10\%) apenas discreto impacto na sua qualidade de vida, apesar do alto valor no índice de incontinência anal. Por outro lado, seis pacientes $(12 \%)$ com incontinência anal moderada ou leve apresentaram grande repercussão na qualidade de vida, especialmente nos domínios depressão e constrangimento. Ressalte-se que esses pacientes negavam qualquer alteração psíquica durante o período em que não apresentavam o sintoma. Essas discrepâncias podem estar relacionadas a fatores sócio-culturais inerentes ao indivíduo e reforçam a importância de associar-se o estudo da qualidade de vida às medidas convencionais, para assim demonstrar a percepção do paciente.

Embora no presente estudo a deterioração da qualidade de vida tenha sido significativamente maior nos pacientes com incontinência anal, observou-se que a qualidade de vida também se encontra afetada nos pacientes com constipação intestinal quando comparada à dos indivíduos voluntários hígidos. Alguns autores, porém, utilizando os instrumentos GIQL - "Gastrintestinal Quality of Life" e IBDQ_ "Inflammatory Bowel Disease Questionnaire", não encontraram diferenças significativas, tanto na comparação da qualidade de vida de incontinentes anais com constipados intestinais, como na comparação com os indivíduos voluntários hígidos ${ }^{(21,30,37)}$. Esses resultados podem ser explicados pelo fato dos questionários acima citados não serem específicos, provavelmente, pouco adequados para o estudo do sintoma abordado ${ }^{(10,15,17)}$.

O constrangimento foi notado em $74 \%$ dos portadores de incontinência anal. Esse dado sugere que muitas vezes o paciente não evita o convívio social somente pela depressão, mas também pelo constrangimento.

A acurácia das propriedades de medida, bem como da sua tradução e adaptação cultural, tornam o questionário um parâmetro adicional importante na avaliação de resultados da qualidade de vida do paciente portador de incontinência anal. A informação que traduz a relação do paciente com sua doença é importante para a postura do médico perante a doença e o doente. Desta forma, ao invés de avaliar apenas os parâmetros clínicos e laboratoriais, obtém-se avaliação mais completa do paciente, o que permite melhor planejamento terapêutico e avaliação a longo prazo.

Portanto, a tradução e a validação do FIQL, instrumento específico de avaliação de qualidade de vida na incontinência anal, será de futura utilidade em ensaios clínicos, para comparação entre dados de diferentes casuísticas e na avaliação da resposta terapêutica. 
Yusuf SAI, Jorge JMN, Habr-Gama A, Kiss DR, Gama-Rodrigues J. Evaluation of quality of life in anal incontinence: validation of the questionnaire FIQL (Fecal Incontinence Quality of Life). Arq Gastroenterol 2004;41(3):202-8.

ABSTRACT - Background - Anal incontinence causes physical and psychological incapacity, determining impact on quality of life. However, there are no specific tools to quantify this impact in our population. Aim - The evaluation of quality of life in anal incontinence, through validation of the FIQL ("Fecal Incontinence Quality of Life Scale"). FIQL is a questionnaire composed of 29 questions, grouped into four domains: lifestyle, behavior, depression and embarrassment. For each question, the scale ranges from 1 to 4, except questions 1 and 4, which ranges from 1-5 and 1-6, respectively. Material and method - FIQL scale underwent both translation and cultural adaptation processes, giving rise to a final Portuguese version. This version was then used in a validation study to test measurement properties (reproducibility and validity). The reproducibility was tested through application of FIQL questionnaire by two observers in 50 patients with anal incontinence. After a period of 7 to 10 days, the questionnaire was applied again by one of the observers. The constructive validity was assessed by correlating the FIQL questionnaire results with both, a generic questionnaire for quality of life (SF-36) and the Jorge-Wexner incontinence score results. The discriminative validity was evaluated comparing the results of the FIQL to incontinence group with two other groups: healthy volunteers and patient with chronic idiopathic constipation. Results - The correlation among results domains of FIQL questionnaire and results of short form-36 and the incontinence score were statistically significant. The quality of life is impaired in all of domains of FIQL for incontinent patients: lifestyle: 2.4 behaviour:2.0, depression:2.5 and embarrassment:1.9, when compared to healthy volunteers (3.9, 3.9, 4.1 and 4.0), and patients with chronic constipation (3.7, 3.8, 3.6 and 3.8), respectively. Conclusion - The FIQL questionnaire is effective and reproducible in its measuring properties, and it can be useful as an instrument to assess quality of life in anal incontinence in our population.

HEADINGS - Anal incontinence. Quality of life. Questionnaire.

\section{REFERÊNCIAS BIBLIOGRÁFICAS}

1. Beck AT, Steer RA. Beck depression inventory. Manual. San Antonio, TX: Psychologica Corporation; 1993

2. Bell MJ, Bombardier C, Tugwell P. Measurement of functional status, quality of life, and utility in rheumatoid arthritis. Arthritis Rheum 1990;33:591-601.

3. Boers M, Tugwell P. The validity of pooled outcome measures (indices) in rheumatoid arthritis clinical trials. J Rheumatol 1993;20:568-74.

4. Bowling A. What things are important in people's lives? A survey of the public's judgements to inform scales of health related quality of life. Soc Sci Med 1995;41:1447-62.

5. Cantarelli FB, Szejnfeld VL, Oliveira LM, Ciconelli RM, Ferraz MB. Quality of life in patients with osteoporosis fractures: cultural adaptation, reability and validity of the Osteoporosis Assessment Questionnaire. Clin Exp Rheumatol 1999;17:547-51.

6. Chassagne P, Landrin I, Neveu C, Czernichow P, Doucet J, Denis P, Bercoff E. Fecal incontinence in the institutionalized elderly: incidence, risk factors and prognosis. Am J Med 1999;106:185-90.

7. Ciconelli RM. Tradução para o português e validação do questionário genérico de avaliação de qualidade de vida "Medical outcomes study 36- item short-form health survey (SF-36)" [tese doutorado]. São Paulo: Escola Paulista de Medicina, Universidade Federal de São Paulo; 1997.

8. Diseth TR, Egeland T, Emblem R. Effects of anal invasive treatment and incontinence on mental health and psychosocial functioning of adolescents with Hirschprung's disease and low anorectal anomalies. J Pediatr Surg 1998;33:468-75.

9. Enck P, Gaber S, Rothmann W, Erckenbrecht JF. Prevalence of fecal incontinence and degree of information possessed by family physician and health insurance. Z Gastroenterol 1991;29:538-40.

10. Eypasch E, Williams JI, Dauphinee WS, Ure BM, Schmulling C, Neugebauer E, Troidl $\mathrm{H}$. Gastrointestinal quality of life index: development, validation and application of a new instrument. Br J Surg 1995;82:216-22.

11. Ferraz MB, Oliveira LM, Araujo PM, Atra E, Tugwell P. Crosscultural reability of the physical ability dimension of the health assessment questionnaire. J Rheumato 1990;17:813-7.

12. Fleck MPA, Louzada S, Xavier M, Chachamovich E, Vieira G, Santos L, Pinzon V. Aplicação da versão em português do instrumento de avaliação de qualidade de vida da Organização Mundial da Saúde (WHOQOL-100). Rev Saúde Pública 1999;33:198-205.

13. Gerin P, Dazord A, Boissel J, Chifflet R. Quality of life assessment in therapeutic trials: rationale for and presentation of a more appropriate instrument. Fundam Clin Pharmacol 1992;6:263-76.

14. Girard M. Episiotomy and faecal incontinence. Lancet 1999;354:2169.

15. Guest M, Smith JJ, Davies AH. Quality of life in patients with benign anorectal disorders. Br J Surg 1999;86:843.

16. Guillemin F, Bombardier C, Beaton D. Cross-cultural adaptation of health-related quality of life measures: literature review and proposed guidelines. J Clin Epidemiol 1993;46:1417-32

17. Guyatt GH, Mitchell A, Irvine EJ, Singer J, Williams N, Goodacre R, Tompkins CA. New measure of health status for clinical trials in inflammatory bowel disease. Gastroenterology 1989;96:804-10
18. Hamilton M. Development of a rating scale for primary depressive illness. Br J Soc Clin Psychol 1967;6:278-96.

19. Huppe D, Enck P, Kruskenper G, May B. [Psychosocial aspects of fecal incontinence]. Leber Magen Darm 1992;22:138-42.

20. Jenkinson C. Evaluating the efficacy of medical treatment possibilities and limitations. Soc Sci Med 1995;41:1395-401.

21. Johanson JF, Lafferty J. Epidemiology of fecal incontinence: the silent affliction. Am J Gastroenterol 1996;91:33-6.

22. Jorge JMN, Wexner SD. Etiology and management of fecal incontinence. Dis Colon Rectum 1993;36:77-97.

23. Koloski NA, Talley NJ, Boyce PM. The impact of functional gastrointestinal disorders on quality of life. Am J Gastroenterol 2000;95:67-71.

24. Lehur AP, Leroi AM. Incontinence anale de l'adulte. Recommandations pour la pratique clinique. Gastroenterol Clin Biol 2000;24:299-414.

25. Leigh RJ, Turnberg LA. Faecal incontinence: the unvoiced symptom. Lancet 1982;1:1349-51.

26. Mellgren A, Jensen LL, Zetterstrom, J.P, Wong DW, Hofmeister JH, Lowry AC. Longterm cost of fecal incontinence secondary to obstetric injuries. Dis Colon rectum 1999; 42: 857-67.

27. O'Brien PE, Stewart S. Restoring control: the action neosphincter. Artificial bowel sphincter in treatment of anal incontinence. Dis Colon Rectum 2000;43:1213-6.

28. Osoba D, Rodrigues G, Myles J, Zee B, Pater J. Interpreting the significance of changes of quality of life scores. J Clin Oncol 1998;16:139-44.

29. Osterberg A, Edebol Eeg-Olofsson K, GrafW. Results of surgical treatment for faecal incontinence. Br J Surg 2000;87:1546-52.

30. Osterberg A, Graf W, Karlbom U, Pahlman L. Evaluation of a questionnaire in the assessment of patients with faecal incontinence and constipation. Scand J Gastroenterol 1996;31:575-80.

31. Patrick DL, Deyo RA. Generic and disease-specific measures in assessing health status and quality of life. Med Care 1989;27:217-32.

32. Perneger TV, Léplege A, Etter JF. Cross-cultural adaptation of a psychometric instrument: two methods compared. J Clin Epidemiol 1999;52:1037-46.

33. Reilly WT, Talley NJ, Pemberton JH, Zinsmeister R. Validation of a questionnaire to assess fecal incontinence and associated risk factors. Dis Colon Rectum 2000;43:146-54

34. Rockwood TH, Church JM, Fleshman JW, Kane RL, Mavratonis C, Wexner SD, Bliss RND, Lowry AC. Patient and surgeon ranking of the severity of symptoms associated with fecal incontinence. Dis Colon Rectum 1999;42:1525-32.

35. Rockwood TH, Church JM, Fleshman JW, Kane RL, Mavrantonis C, Thorson AG, Lowry AC. Fecal incontinence quality of life scale: quality of life instrument for patients with fecal incontinence. Dis Colon Rectum 2000;43:9-17.

36. Rothbath J, Bemelman WA, Meijerink HJ, Stiggelbout AM, Delemarre BVM. What is the impact of fecal incontinence on quality of life? Dis Colon Rectum 2001:44:67-71.

37. Sailer M, Bussen D, Debus ES, Fuchs KH, Thiede A. Quality of life in patients with benign anorectal disorders. Br J Surg 1998;85:1716-9.

38. Sailer M, Bussen D, Fuchs HK, Thiede A. Quality of life in patients with faecal incontinence. Langenbecks Arch Chir 1998 Supp II:973-5.

39. Sangwan YP, Coller JA. Fecal incontinence. Surg Clin North Am 1998;4:1716-9. 
40. Sharpe A, Read A, Slater B, Varma J. Quality of life assessment in patients with faecal incontinence. Int J Colorect Dis 1997;12:124-7.

41. Soffer EE, Hull T. Fecal incontinence: a practical approach to evaluation and treatment. Am J Gastroenterol 2000;95:1873-80.

42. Sullivan M. Cross-cultural validation for quality of life. J Clin Epidemiol $1994 ; 47: 1465$.

43. Testa MA, Simonsen DC. Assessment of quality of life outcomes. N Engl J Med 1996;334:835-40.
44. Tugwell P, Bombardier CA. Methodologic framework for developing and selecting endpoints in clinical trials. J Rheumatol 1982;9:758-62.

45. Ware JE, Sherbourne CD. The MOS 36-item short-form heath survey (SF-36): conceptual framework and item selection. Med Care 1992;30:473-83.

Recebido em 7/10/2003. Aprovado em 19/3/2004. 